\title{
Visual Acuity, Contrast Sensitivity and Color Vision Three Years After Iodine-125 Brachytherapy for Choroidal and Ciliary Body Melanoma
}

\author{
Irena Tsui ${ }^{1}$, Robert M. Beardsley ${ }^{1}$, Tara A. McCannel ${ }^{1}$, Scott C. Oliver ${ }^{2}$, Melissa W. Chun ${ }^{1}$, \\ Steve P. Lee ${ }^{3}$, Phillip E. Chow $^{3}$, Nzhde Agazaryan ${ }^{3}$, Fei Yu ${ }^{1}$ and Bradley R. Straatsma ${ }^{*}, 1$ \\ ${ }^{1}$ Department of Ophthalmology and Jules Stein Eye Institute, University of California, Los Angeles, Los Angeles, CA, USA \\ ${ }^{2}$ Department of Ophthalmology, University of Colorado, Denver, Aurora, CO, USA \\ ${ }^{3}$ Department of Radiation Oncology, University of California, Los Angeles, Los Angeles, CA, USA
}

\begin{abstract}
Purpose: To report visual acuity, contrast sensitivity and color vision prior to, 1 year after, 2 years after and 3 years after iodine-125 brachytherapy for choroidal and ciliary body melanoma (CCM).

Design: Prospective interventional case series.

Participants: Thirty-seven patients (37 eyes) with CCM.

Methods: Patients had best-corrected Early Treatment Diabetic Retinopathy Study (ETDRS) visual acuity, Pelli-Robson contrast sensitivity and Hardy-Rand-Rittler color vision measurement; comprehensive ophthalmology examination; optical coherence tomography; and ultrasonography at baseline prior to, 1 year after, 2 years after and 3 years after I- 125 brachytherapy.

Main Outcome Measures: Visual acuity, contrast sensitivity and color vision prior to, 1 year after, 2 years after and 3 years after brachytherapy.

Results: Nineteen (19) men and 18 women with mean age of 58 years (SD 13, range 30-78) prior to, 1 year after, 2 years after and 3 years after brachytherapy had mean best-corrected visual acuity of 77 letters (20/32), 65 letters (20/50), 56 letters (20/80) and 47 letters (20/125); contrast sensitivity of 30, 26, 22 and 19 letters; color vision of 26, 20, 17 and 14 test figures, respectively. Decrease in visual acuity, contrast sensitivity and color vision was statistically significant from baseline at 1 year, 2 years, and 3 years after brachytherapy. Decreased acuity at 3 years was associated with mid-choroid and macula melanoma location, $\geq 4.1 \mathrm{~mm}$ melanoma height, radiation maculopathy and radiation optic neuropathy.

Conclusion: 1, 2 and 3 years after brachytherapy, eyes with CCM had significantly decreased visual acuity, contrast sensitivity and color vision.
\end{abstract}

Keywords: Brachytherapy, choroidal melanoma, ciliary body melanoma, contrast sensitivity, visual acuity.

\section{INTRODUCTION}

Choroidal and ciliary body melanoma (CCM) is the most common primary intraocular malignancy in adults. Overall incidence of uveal melanoma is reported as approximately 5.1 per million per year in the United States [1].

The Collaborative Ocular Melanoma Study (COMS) randomized clinical trial of iodine-125 (I-125) brachytherapy versus enucleation for medium-sized choroidal melanoma $(2.5-10.0 \mathrm{~mm}$ in thickness and $\leq 16.0 \mathrm{~mm}$ in diameter) showed that, for patients who met the eligibility criteria, there was no statistically significant difference in all-cause mortality between I-125 brachytherapy and enucleation 5, 10 and 12 years following treatment [2-4].

*Address correspondence to this author at the Jules Stein Eye Institute, 100 Stein Plaza, UCLA, Los Angeles, CA 90095-7000, USA; Tel: (310) 8255051; Fax: (310) 206-4293; E-mail: straatsma@jsei.ucla.edu
The COMS trial supported the use of globe-conserving I125 brachytherapy. However, adverse effects of I-125 brachytherapy included dry eye and keratopathy, iris neovascularization and glaucoma, radiation cataract, radiation maculopathy and radiation optic neuropathy [5-11]. Reflecting these adverse effects, the COMS reported median visual acuity following I-125 brachytherapy for mediumsized choroidal melanoma in 623 patients (623 eyes) as $20 / 32$ at baseline prior to brachytherapy and 20/125 3 years after brachytherapy [5].

This current study was carried out, in accordance with a previously reported protocol [12], to further evaluate the effect of I-125 brachytherapy for CCM on central visual function by measurement of best-corrected visual acuity, contrast sensitivity and color vision prior to, 1 year after, 2 years after, and 3 years after brachytherapy. 


\section{MATERIALS AND METHODOLOGY}

This prospective interventional case study was conducted in accordance with the Declaration of Helsinki and approved by the Institutional Review Board of the University of California, Los Angeles (UCLA). Informed consent was obtained from all patients and the study adhered to provisions of the United States Health Insurance Portability and Accountability Act of 1996.

Adult patients with CCM undergoing I-125 brachytherapy were eligible to enroll unless there was ocular disease unrelated to melanoma affecting central visual function of either eye, prior radiation therapy to the head or medical condition likely to interfere with 3 year follow-up. Baseline evaluation was performed $\leq 30$ days prior to brachytherapy and post-treatment evaluation was carried out 1 year, 2 years and 3 years after brachytherapy. Pretreatment and post-treatment evaluation included protocol refraction immediately prior to each visual acuity, contrast sensitivity and color vision measurement; comprehensive ophthalmic examination; fundus photography; optical coherence tomography; and ultrasonography. Fluorescein angiography was performed at baseline and as clinically indicated thereafter.

Visual Acuity. After refraction performed in accordance with the COMS Protocol [13], best-corrected visual acuity was measured as the number of letters read with each eye at $3.2 \mathrm{~m}$ (or at $1.6 \mathrm{~m}$ or $0.8 \mathrm{~m}$ if necessary) using the FerrisBailey Early Treatment Diabetic Retinopathy Study (ETDRS) visual acuity chart (The Lighthouse, Inc., Long Island City, NY) with standardized lighting. Snellen equivalent acuities of the ETDRS score were calculated from COMS Chart 02 [13].

Contrast Sensitivity. Contrast sensitivity was measured as the number of letters with decreasing contrast recognized with each eye on a standard luminance background $[12,13]$. Contrast threshold was assessed at one meter with the PelliRobson Contrast Sensitivity Chart (Clement Clark, Dayton, $\mathrm{OH}$ ), using standardized lighting and $+0.75 \mathrm{D}$ add over the distance refraction. For acuities below 20/320, the chart distance was shortened so that the letters appeared at twice the acuity threshold.

Color Vision. Hardy-Rand-Rittler Pseudoisochromatic Color Test Plates, 4th Edition (Richmond Products, Inc., Albuquerque, NM), were used to assess color vision in each eye with standardized illumination at $0.75 \mathrm{~m}$. and $+1.25 \mathrm{D}$ add over the distance vision. Correct responses to 28 diagnostic figures were scored.

Iodine-125 Brachytherapy. I-125 brachytherapy for CCM has been described elsewhere [14, 15]. Briefly, melanomas were treated to a target dose of $85 \mathrm{~Gy}$ at the tumor apex. Gold plaques consistent with COMS standards covered the tumor base and a surrounding margin of $3 \mathrm{~mm}$. Under monitored local anesthesia and akinesia achieved with retrobulbar block, partial or 360-degree conjunctival peritomy was performed, rectus muscles were isolated and detached with a hang-back suture when required for plaque placement. Tumor borders were localized with transillumination and confirmed with indirect ophthalmoscopy. Transscleral fine needle aspiration biopsy was performed for cytopathology and cytogenetic study. The plaque was secured in position with 5-0 Mersilene sutures and placement was confirmed with intraoperative B-scan ultrasonography. The plaque remained in place for the duration determined by radiation dosimetry calculations and was removed under monitored local anesthesia.

Statistical Analysis. Statistical analysis used SAS version 9.1 (Cary, NC). Difference in visual acuity, contrast sensitivity and color vision from baseline to 1 year, 2 years, and 3 years after brachytherapy, and difference in visual acuity from baseline to three years for melanoma location, melanoma thickness, cataract, maculopathy and optic neuropathy were assessed with the Wilcoxon signed rank test. Association of maculopathy and optic neuropathy with radiation dose was evaluated with the Fisher exact test and the Kruskal-Wallis test. P-value $<0.05$ was considered statistically significant.

\section{RESULTS}

Thirty-seven patients (37 eyes) with CCM (TNIM Classification T2a and T2b) [16] consisted of 19 (52\%) men and $18(48 \%)$ women with mean age at brachytherapy of 58 years (SD 13 years, range 30-78 years). Ethnicity was White $33(90 \%)$, Hispanic $2(5 \%)$, and Asian $2(5 \%)$. There were 19 left eyes and 18 right eyes.

For all $\mathrm{CCM}$ eyes at baseline prior to, 1 year after, 2 years after, and 3 years after brachytherapy, mean visual acuity was 77 letters (20/32), 65 letters (20/50), 56 letters $(20 / 80)$ and 47 letters (20/125); contrast sensitivity was 30 letters, 26 letters, 22 letters and 19 letters; color vision was 26 figures, 20 figures, 17 figures and 14 figures, respectively (Table 1). Decrease in visual acuity, contrast sensitivity and color vision from baseline at 1 year, 2 years and 3 years was statistically significant (Table $\mathbf{1}$ ).

Furthermore, 1 year after, 2 years after and 3 years after brachytherapy, change in visual acuity was statistically significantly correlated with change in contrast sensitivity (Spearman correlation coefficient $=0.87 \quad(\mathrm{p}<0.0001), 0.91$ $(\mathrm{p}<0.0001)$ and $0.90(\mathrm{p}<0.0001)$ respectively $)$ and change in color vision (Spearman correlation coefficient $=0.50$ $(p=0.005), \quad 0.79 \quad(p=<0.0001) \quad$ and $0.78 \quad(p<0.0001)$ respectively). Therefore, decrease in visual acuity, contrast sensitivity and color vision were significantly correlated and generally declined simultaneously after brachytherapy.

At baseline, visual acuity was $\geq 20 / 20$ in 15 (41\%) eyes and $\leq 20 / 200$ in $2(5 \%)$ eyes. Three years after brachytherapy, visual acuity was $\geq 20 / 20$ in $4(11 \%)$ eyes and $\leq 20 / 200$ in 15 (41\%) eyes (Table 1).

Analysis of CCM location and visual acuity showed location-dependent vision loss. At baseline and 3 years after brachytherapy, ciliary body/anterior (pre-equatorial) choroid tumors $(\mathrm{n}=8)$ had a mean visual acuity of 76 letters and 77 letters respectively, mid-choroid tumors $(\mathrm{n}=16) 85$ letters and 44 letters, and macula tumors $(\mathrm{n}=13) 66$ letters and 32 letters (Table 2). The decrease in visual acuity for ciliary body/anterior choroid tumors was not significant $(\mathrm{p}=0.41)$, whereas mid-choroid and macula tumors had statistically significant change in visual acuity $(\mathrm{p}=0.002$ and 0.006 respectively). 
Table 1. Visual acuity, contrast sensitivity and color vision three years after iodine-125 brachytherapy.

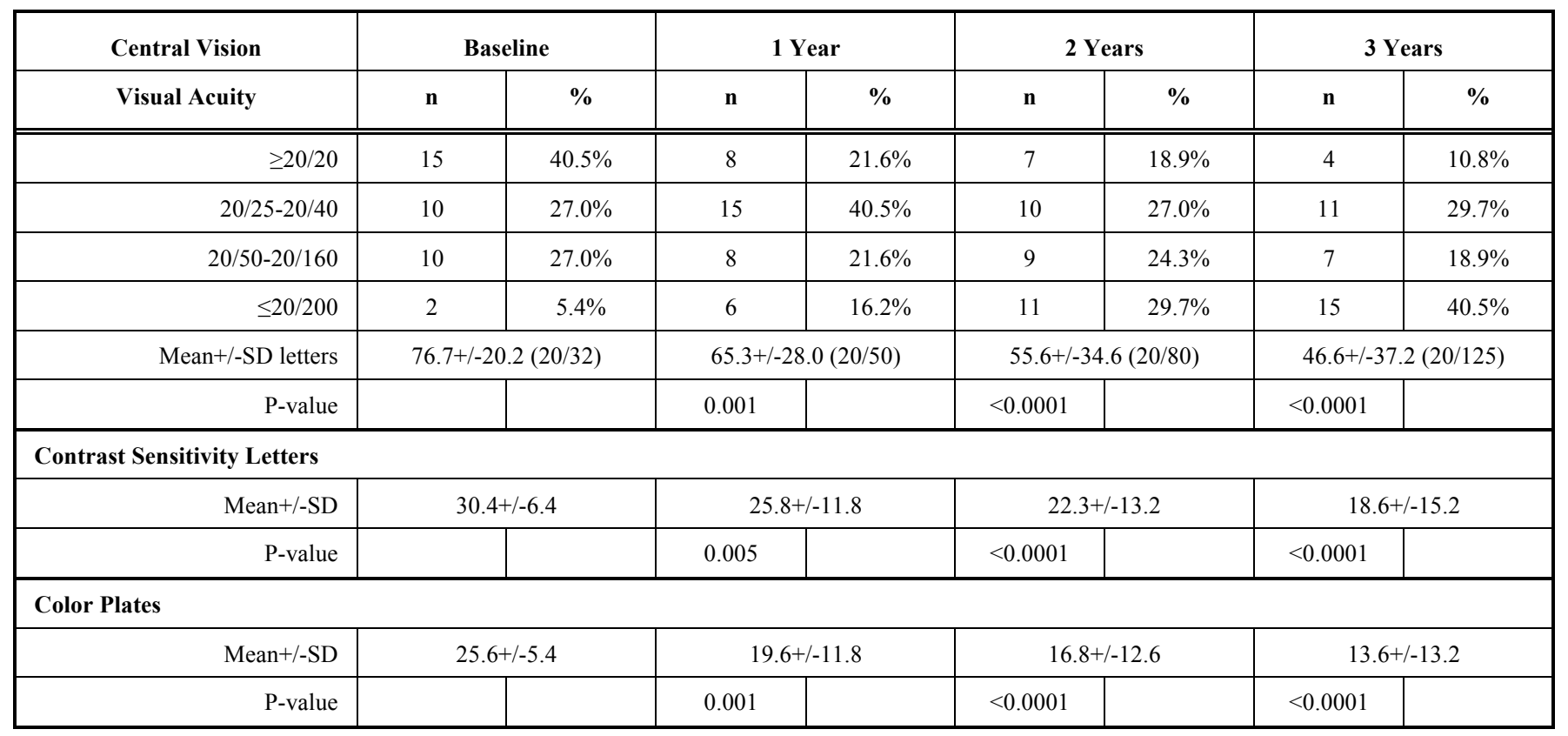

Melanoma apical height and visual acuity loss showed a significant correlation for thicker tumors. Melanomas with apical height of $\leq 4.0 \mathrm{~mm}(\mathrm{n}=21)$ had a mean visual acuity decrease from 79 letters to 58 letters, a decrease of borderline statistical significance $(\mathrm{p}=0.051)$, whereas melanomas $\geq 4.1 \mathrm{~mm}$ in apical height $(\mathrm{n}=16)$ had a mean decrease from 74 letters to 35 letters, a decrease that was statistically significant $(\mathrm{p}<0.0001$, Table 2$)$.

Radiation Cataract, Maculopathy and Optic Neuropathy. Radiation cataract, defined as visually significant progression of $2+$ or greater lens opacity compared to the fellow eye, developed in $13(41 \%)$ of 32 eyes phakic at time of brachytherapy and was associated with significant decrease in mean visual acuity from 72 letters at baseline to 31 letters 3 years after brachytherapy $(p=0.008)$. This decrease in visual acuity in eyes with cataract was somewhat greater than the decrease in eyes aphakic at baseline or without cataract 3 years after brachytherapy, but the difference was not statistically significant $(p=0.36)$. This is consistent with the practice of cataract surgery when clinically indicated.
Radiation maculopathy was identified by clinical abnormalities and, when indicated, by fluorescein angiography in $21(57 \%)$ of 37 eyes and associated with a significant decrease in mean visual acuity from 72 letters at baseline to 27 letters 3 years after brachytherapy $(p<0.0001)$.

Radiation optic neuropathy was diagnosed on the basis of optic disc pallor in $4(11 \%)$ of 37 eyes and associated with severe decrease in vision from 45 letters at baseline to $<1$ letter 3 years after brachytherapy, a decrease that was clinically but not statistically significant in this limited sample $(\mathrm{p}=0.13)$.

Fellow Eye Visual Function. The 37 unaffected fellow eyes had a baseline mean best-corrected visual acuity of 90 letters (20/16), contrast sensitivity of 35 letters, and color vision of 28 figures, measurements that were significantly better than the corresponding baseline measurements in CCM eyes $(\mathrm{p}<0.0001,0.0001$ and 0.005 respectively). Three years after brachytherapy, mean best-corrected visual acuity in fellow eyes remained at 90 letters $(20 / 16)$.

Table 2. Melanoma location, melanoma apical height and visual acuity outcome.

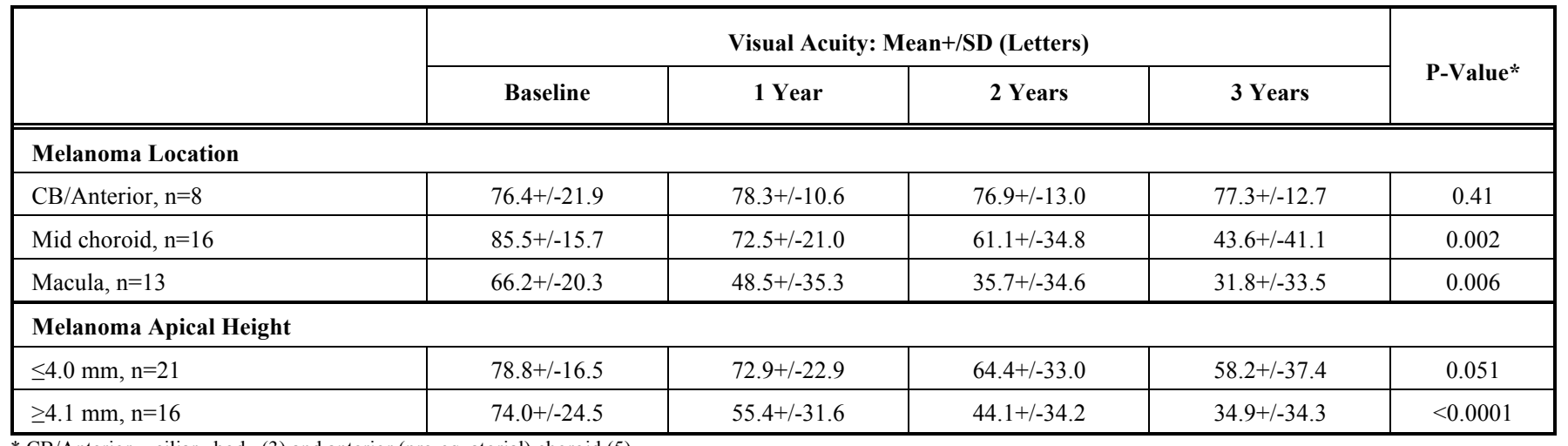

$* \mathrm{CB} /$ Anterior $=$ ciliary body (3) and anterior (pre-equatorial) choroid (5). 


\section{DISCUSSION}

This study of best-corrected visual acuity, contrast sensitivity and color vision prior to, 1 year after, 2 years after, and 3 years after globe-conserving I-125 brachytherapy for CCM provided new information regarding important parameters of brachytherapy-related vision impairment, confirmed major risk factors for brachytherapy-related vision loss and compared vision parameters in CCM eyes with corresponding measurements in unaffected fellow eyes.

For the first time, to our knowledge, contrast sensitivity and color vision measurement were systematically combined with assessment of best-corrected visual acuity to demonstrate the extent of central vision impairment following brachytherapy for uveal melanoma. Snellen visual acuity alone is insufficient for full understanding of visual performance. Decrease in contrast sensitivity and color perception measure distinctly different properties of vision and combine with impairment of visual acuity to interfere with activities of daily living such as mobility at low levels of illumination, perception of stairs and curbs, driving, reading, using tools and sewing [17-19]. This multi-faceted impairment of vision is particularly significant in patients with CCM and brachytherapy in the better-seeing eye and in patients with subsequent loss of vision in the better-seeing fellow eye.

Of practical importance, change in visual acuity, contrast sensitivity and color vision was statistically significantly correlated at 1 year, 2 years and 3 years after brachytherapy. Thus, visual acuity, contrast sensitivity and color vision generally declined simultaneously in the period after brachytherapy.

Concerning prognosis, this study confirmed several risk factors for brachytherapy-related vision loss reported in other studies $[5,6,20,21]$. For example, this study of 37 patients (37 eyes) with CCM treated with I-125 brachytherapy reported mean visual acuity at baseline, 1 year, 2 years, and 3 years after brachytherapy as 20/32, 20/50, 20/80 and 20/125 respectively (Table 1 ). The COMS report of 623 patients (623 eyes) with choroidal melanoma treated with I125 brachytherapy described median visual acuity at baseline, 1 year, 2 years, and 3 years after brachytherapy as $20 / 32,20 / 40,20 / 50$ and 20/125 respectively. In addition, this study and other reports identified greater risk of visual acuity loss associated with mid-choroid or macula tumor location, large tumor size, radiation maculopathy and radiation optic neuropathy (Table 2). These findings of visual acuity loss and relative risk for severe impairment of vision are useful in patient counseling and follow-up care. However, it should be noted that individual patients have a wide range of vision loss in response to brachytherapy and counseling should recognize individual variability.

Finally, comparison of the CCM eyes with fellow eyes in this study identified decrease in visual acuity, contrast sensitivity and color vision in CCM eyes prior to brachytherapy. Thus, impairment of central vision in melanoma eyes after brachytherapy is the result of both melanoma-related and brachytherapy-related central vision loss. Retention of normal vision in the fellow eyes 3 years after brachytherapy generally mitigated the functional impact of vision loss in the melanoma eyes and emphasized the importance of ophthalmologic care for the fellow eyes.

Relative strengths for this study of central vision in CCM eyes treated with brachytherapy are prospective design, protocol refraction prior to each assessment of central vision; measurement of best-corrected visual acuity, contrast sensitivity and color vision at baseline prior to, 1 year after, 2 years after, and 3 years after brachytherapy; correlation with melanoma size and location; and corresponding measurements of central vision in the fellow eyes. Relative weakness is the limited sample size of 37 patients.

In summary, this study of 37 patients (37 eyes) with choroidal and ciliary body melanoma treated with I-125 brachytherapy and followed for 3 years showed statistically significant and progressive mean decrease of best-corrected visual acuity, contrast sensitivity and color vision in the treated eyes. Greater decrease in visual acuity 3 years after brachytherapy was correlated with mid-choroidal and macula tumor location, baseline tumor height of $\geq 4.1 \mathrm{~mm}$, radiation maculopathy and radiation optic neuropathy.

\section{CONFLICT OF INTEREST}

The authors confirm that this article content has no conflict of interest.

\section{ACKNOWLEDGEMENTS}

Authors acknowledge support by Research to Prevent Blindness Unrestricted Grant and the George and Ruth E. Moss Trust.

\section{REFERENCES}

[1] Singh AD, Turell MD, Topham AK. Uveal Melanoma: Trends in incidence, treatment, and survival. Ophthalmology 2011; 118: 1881-5.

[2] Diener-West M, Earle JD, Fine SL, et al. The COMS randomized trial of iodine 125 brachytherapy for choroidal melanoma. III. Initial mortality findings. COMS report no. 18. Arch Ophthalmol 2001; 119: 969-82.

[3] Collaborative Ocular Melanoma Study Group. Ten-year follow-up of fellow eyes of patients enrolled in Collaborative Ocular Melanoma Study randomized trials: COMS report no. 22. Ophthalmology 2004; 111: 966-76.

[4] Collaborative Ocular Melanoma Study Group. The COMS randomized trial of iodine 125 brachytherapy for choroidal melanoma. V. Twelve-year mortality rates and prognostic factors. COMS report no. 28. Arch Ophthalmol 2006; 123: 1684-93.

[5] Melia BM, Abramson DH, Albert DM, et al. Collaborative Ocular Melanoma Study (COMS) randomized trial of I-125 brachytherapy for medium choroidal melanoma. I. Visual acuity after 3 years. COMS report no. 16. Ophthalmology 2001; 108: 348-66.

[6] Wen JC, Oliver SC, McCannel TA. Ocular complications following I-125 brachytherapy for choroidal melanoma. Eye 2001; 23: 1254-68.

[7] Detorakis ET, Engstrom Jr RE, Wallace R, et al. Iris and anterior chamber angle neovascularization after iodine 125 brachytherapy for uveal melanoma. Ophthalmology 2005; 112(3): 505-10.

[8] Collaborative Ocular Melanoma Study Group. Incidence of cataract and outcomes after cataract surgery in the first 5 years after Iodine 125 brachytherapy in the Collaborative Ocular Melanoma Study. COMS report no 27. Ophthalmology 2007; 114(7): 1363-71.

[9] Horgan N, Shields CL, Mashayekhi A, et al. Classification and treatment of radiation maculopathy. Curr Opin Ophthalmol 2010; 21: 233-8.

[10] Mukai S, Guyer DR, Gragoudas ES. Radiation retinopathy. In: Albert DM, Jakobiec FA, Eds. Principles and practices of 
ophthalmology: clinical practice W.B. Saunders Company Pennsylvania 1994; vol. 2: pp. 1038-41.

[11] Kim IK, Lane AM, Egan KM, et al. Natural history of radiation papillopathy after proton beam irradiation of parapapillary melanoma. Ophthalmology 2010; 117(8): 1617-22.

[12] Oliver SCN, Young TA, Kobe LH, et al. Assessment of central vision and macular structure in patients undergoing iodine-125 brachytherapy for ciliochoroidal melanoma. Am J Clin Oncol 2008; 31(5): 488-92.

[13] Collaborative Ocular Melanoma Study Group. COMS manual of procedures. Virginia: National Technical Information Service 1995.

[14] Young TA, Rao NP, Glasgow BJ, et al. Fluorescent in situ hybridization for monosomy 3 via 30 -gauge fine-needle aspiration biopsy of choroidal melanoma in vivo. Ophthalmology 2007; 114(1): 142-6.

[15] McCannel TA, Burgess LB, Rao NP, et al. Identification of candidate tumor oncogenes by integrative molecular analysis of choroidal melanoma fine needle aspiration biopsy specimens. Arch Ophthalmol 2010; 128(9): 1170-7.
[16] American Joint Committee on Cancer cancer staging manual, $7^{\text {th }}$ ed. New York Dordrecht Heidelberg London: Springer 2010; pp. 547-53.

[17] Rubin GS, Bandeen-Roche K, Huang GH, et al. The association of multiple visual impairments with self-reported visual disability: SEE project. Invest Ophthalmol Vis Sci 2001; 42(1): 64-72.

[18] Arden GB. The importance of measuring contrast sensitivity in cases of visual disturbance. Br J Ophthalmol 1978; 62(4): 198-209.

[19] Colenbrander A, Fletcher DC. Beyond BCVA: Thorough vision assessment for retinal physicians Retinal Physician November December 2011; 50-55.

[20] Finger PT, Chin KJ, Guo-pei Y, et al. Risk factors for radiation maculopathy after ophthalmic plaque radiation for choroidal melanoma. Am J Ophthalmol 2010; 149(4): 608-15.

[21] Sagoo MS, Shields CL, Emrich J, et al. Plaque radiotherapy for juxtapapillary choroidal melanoma; treatment complications and visual outcomes in 650 consecutive cases. JAMA Ophthalmol 2014; 132(6): 697-702. 